\title{
Heterosis Studies in CMS Based and Conventional Hybrids for Yield and Yield Contributing Traits in Cotton (G. hirsutum L.) Over the Environments
}

\author{
G. R. Gopal, D. B. Deosarkar and V. N. Chinchane* \\ Department of Agricultural Botany, (Genetics and Plant Breeding) \\ Vasantrao Naik Marathawada Krishi Vidyapeeth, Parbhani. 431402 (M.S.), India \\ *Corresponding author
}

\begin{tabular}{|c|c|}
\hline & A B S T R A C T \\
\hline $\begin{array}{l}\text { Ke y w o r d s } \\
\text { Heterosis, CMS, } \\
\text { Conventional, } \\
\text { Cotton, Yield }\end{array}$ & \multirow{3}{*}{$\begin{array}{l}\text { The present study comprised of three male sterile CMS lines, three } \\
\text { maintainer lines and ten male fertile males (testers/ restorers) with three } \\
\text { standard checks in two sets i.e. CMS and conventional thus making } 60 \mathrm{~F}_{1} \mathrm{~S} \\
\text { ( } 30 \mathrm{CMS} \text { and } 30 \text { conventional) using Line x Tester mating design. The } \\
\text { crosses, CAK 53A x AKH- 07R, CAK 23B x AKH-07R, CAK 23B x DHy- } \\
286-1 \mathrm{R} \text {, CAK 23B x R-2000- } 23 \text { and SRT-1A x R-2000-26 were found } \\
\text { better for seed cotton yield per plant and hold promise for further } \\
\text { evaluation and commercial exploitation of heterosis both in CMS and } \\
\text { conventional system for high yield. }\end{array}$} \\
\hline Article Info & \\
\hline $\begin{array}{l}\text { Accepted: } \\
\text { 05 February } 2020 \\
\text { Available Online: } \\
10 \text { March } 2020\end{array}$ & \\
\hline
\end{tabular}

\section{Introduction}

Cotton is one of the most important fiber and cash crop of India and plays a dominant role in the industrial and agricultural economy of the country. It provides the basic raw material (cotton fibre) to cotton textile industry. In India, most of the cotton hybrids are produced manually by using conventional method of hand emasculation and pollination thus making hybrid seed production expensive. In the recent years, the phenomenon of male sterility being used for heterosis breeding which has helped to reduce cost of hybrid seed production about 70 per cent (Meshram et al., 1995). In cotton, different sources of male sterility are available. Out of which genetic male sterility (GMS) and cytopolasmic genetic male sterility (CGMS) are mainly used in both diploid and tetraploid species of cotton for hybrid development. Male sterility based cotton hybrids are blessed with cheaper and higher seed production. But both the male sterility system (GMS and CGMS) have low productivity than their fertile counterpart. Low productivity of lines developed from sterile cytoplasm in case of CGMS system may be explained with 
Plasmon sensitivity hypothesis, according to which the maternal cytoplasm provides an unstable substrate from paternal genes.

Exploitation of hybrid vigour in cotton has gained much significance in view of its tremendous yield increase and is considered as outstanding accomplishment of plant breeding. There is a continuous need to evolve new hybrids which should exceed the existing hybrids in yield and fibre quality. Breeding of commercial hybrids has been proved to be the most important tool in increasing the yields of crop plants substantially within a short period.

\section{Materials and Methods}

The present study comprised of three male sterile CMS lines, three maintainer lines and ten male fertile males (testers/ restorers) with three standard checks in two sets i.e. CMS and conventional thus making $60 \quad \mathrm{~F}_{1} \mathrm{~s}$ (30 CMS and 30 conventional) using Line $\mathrm{x}$ Tester mating design. These lines, testers and hybrids along with three checks were sown during kharif, 2016 at three locations viz., Cotton Research Station, Nanded (L-1), Experimental farm of Department of Agricultural Botany, VNMKV, Parbhani (L2) and Experimental farm, Agricultural Research Station, Badnapur (L-3). Observations were recorded on randomly selected five competitive plants from each replication for each genotype on days to $50 \%$ flowering, plant height $(\mathrm{cm})$, number of sympodia per plant, days to $50 \%$ boll burst, earliness index, number of sympodia per plant, number of boll per plant, boll weight $(\mathrm{g})$, seed cotton yield per plant $(\mathrm{g})$, seed index $(\mathrm{g})$, lint index (g) and harvest index.

\section{Results and Discussion}

The experimental results revealed that the heterotic effect was high in the conventional than CMS for the traits like, seed cotton yield per plant, days to $50 \%$ flowering, plant height, boll weight and seed index. But among both the sets of crosses (thirty CMS and thirty conventional) the CMS cross CAK 53A $x$ AKH $07 \mathrm{R}$ possessed the highest heterosis over better parent and standard heterosis for the traits earliness index and lint index. For seed cotton yield per plant the conventional hybrids 21, 3, 18 and 18 and in CMS 13, 2, 10 and 12 exhibited the positive significant heterosis over the better parent, PKV-Hy-4, NHH-206 and NHH-44 respectively. Maximum positive significant crosses were found in conventional system than in CMS system. Similar results of significant desirable heterosis for seed cotton yield and related traits were also reported by Deosarkar et al., (2009), Tuteja and Agrawal (2014) and Sharma et al., (2016).

In the CMS crosses, magnitude of heterosis was high over the better parent and standard check for the characters viz., seed cotton yield per plant, days to $50 \%$ flowering, plant height, number of monopodia per plant, number of bolls per plant, number of sympodia per plant, boll weight, harvest index and moderate for the trait earliness. Heterosis for seed cotton yield in CMS based hybrids have been reported by Tuteja et al., (2011), Shinde et al., (2012), Tuteja and Agrawal (2014).

Among the conventional crosses, higher heterotic effects were found for the traits viz., seed cotton yield per plant, number of monopodia per plant, seed index, number of sympodia per plant, number of bolls per plant and seed cotton yield per plant. Moderately observed for lint index and earliness index. Low heterotic effects were found for days to $50 \%$ boll burst. Heterosis for different traits in conventional hybrids were also reported by workers Deosarkar et al., (2009), Patel et al., (2014), Pushpam et al., (2015) and Sharma et al., (2016). 
Table.1 Per cent heterosis pooled over environments over better parent (BPH), check hybrids PKV-Hy-4 (SH1), NHH-206 (SH 2) and NHH-44 (SH 3)

\begin{tabular}{|c|c|c|c|c|c|c|c|c|c|}
\hline \multirow{2}{*}{$\begin{array}{l}\text { Sr. } \\
\text { No. }\end{array}$} & \multirow[t]{2}{*}{ Crosses } & \multicolumn{4}{|c|}{ Days to $50 \%$ flowering } & \multicolumn{4}{|c|}{ Plant height (cm) } \\
\hline & & ВPH & SH 1 & SH2 & SH3 & ВPH & SH 1 & SH2 & SH3 \\
\hline 1 & CAK 53A X R-42-8 & -1.00 & $2.84 *$ & $3.65 * *$ & $6.99 * *$ & 6.08 & $-7.76 *$ & -1.79 & 0.54 \\
\hline 2 & CAK 53A X R-2000-23 & $-8.71 * *$ & $-5.17 * *$ & $-4.43 * *$ & -1.34 & $-7.60 *$ & $-12.79 * *$ & $-7.14 *$ & -4.94 \\
\hline 3 & CAK 53A X R- 2000-17-2 & $-18.91 * *$ & $-15.76 * *$ & $-15.10 * *$ & $-12.37 * *$ & 5.25 & -6.67 & -0.63 & 1.73 \\
\hline 4 & CAK 53A X R-2000-26 & $-21.89 * *$ & $-18.86 * *$ & $-18.23 * *$ & $-15.59 * *$ & -2.14 & $-8.19 *$ & -2.25 & 0.07 \\
\hline 5 & CAK 53A X R-1044-13 & $-7.71 * *$ & $-4.13 * *$ & $-3.39 * *$ & -0.27 & 4.02 & $-9.55 * *$ & -3.70 & -1.42 \\
\hline 6 & CAK 53A X R-2000-21 & $-8.21 * *$ & $-4.65 * *$ & $-3.91 * *$ & -0.81 & 4.07 & $-9.50 * *$ & -3.65 & -1.36 \\
\hline 7 & CAK 53A X R-23 & $-7.21 * *$ & $-3.62 * *$ & $-2.86 *$ & 0.27 & 0.95 & -1.85 & 4.51 & 6.98 \\
\hline 8 & CAK 53A X R-53 & $-16.42 * *$ & $-13.18 * *$ & $-12.50 * *$ & $-9.68 * *$ & 2.34 & $-6.90 *$ & -0.87 & 1.48 \\
\hline 9 & CAK 53A X AKH-07R & $-7.71 * *$ & $-4.13 * *$ & $-3.39 * *$ & -0.27 & 3.59 & $-9.92 * *$ & -4.09 & -1.82 \\
\hline 10 & CAK 53A X DHY-286-1R & $-21.34 * *$ & $-18.09 * *$ & $-17.45 * *$ & $-14.78 * *$ & 1.32 & $-11.90 * *$ & -6.19 & -3.97 \\
\hline 11 & CAK 53B X R-42-8 & $-10.20 * *$ & $-6.72 * *$ & $-5.99 * *$ & $-2.96 *$ & $7.83 *$ & -6.24 & -0.17 & 2.20 \\
\hline 12 & CAK 53B X R-2000-23 & $-9.20 * *$ & $-5.68 * *$ & $-4.95 * *$ & -1.88 & -2.30 & $-7.79 *$ & -1.82 & 0.51 \\
\hline 13 & CAK 53B X R- 2000-17-2 & $-15.92 * *$ & $-12.66 * *$ & $-11.98 * *$ & $-9.14 * *$ & 4.68 & $-7.17 *$ & -1.16 & 1.18 \\
\hline 14 & CAK 53B X R-2000-26 & $-13.68 * *$ & $-10.34 * *$ & $-9.64 * *$ & $-6.72 * *$ & 0.47 & -5.73 & 0.37 & 2.75 \\
\hline 15 & CAK 53B X R-1044-13 & 1.24 & $5.17 * *$ & $5.99 * *$ & $9.41 * *$ & 1.82 & $-11.47 * *$ & -5.74 & -3.50 \\
\hline 16 & CAK 53B X R-2000-21 & $-6.97 * *$ & $-3.36 * *$ & $-2.60 *$ & 0.54 & 0.51 & $-12.60 * *$ & -6.94 & -4.74 \\
\hline 17 & CAK 53B X R-23 & $-11.19 * *$ & $-7.75 * *$ & $-7.03 * *$ & $-4.03 * *$ & $-8.38 *$ & $-10.92 * *$ & -5.15 & -2.91 \\
\hline 18 & CAK 53B X R-53 & $-13.43 * *$ & $-10.08 * *$ & $-9.38 * *$ & $-6.45 * *$ & -1.12 & $-10.04 * *$ & -4.22 & -1.95 \\
\hline 19 & CAK 53B X AKH-07R & $-9.20 * *$ & $-5.68 * *$ & $-4.95 * *$ & -1.88 & 7.25 & $-6.74 *$ & -0.70 & 1.65 \\
\hline 20 & CAK 53B X DHY-286-1R & $-19.60 * *$ & $-16.28 * *$ & $-15.63 * *$ & $-12.90 * *$ & -2.86 & $-15.53 * *$ & $-10.06 * *$ & $-7.93 *$ \\
\hline 21 & CAK 23A X R-42-8 & $-13.64 * *$ & $-11.63 * *$ & $-10.94 * *$ & $-8.06 * *$ & -1.40 & $-17.96 * *$ & $-12.65 * *$ & $-10.58 * *$ \\
\hline 22 & CAK 23A X R-2000-23 & $-10.70 * *$ & $-7.24 * *$ & $-6.51 * *$ & $-3.49 * *$ & -5.47 & $-10.78 * *$ & -50 & -2.75 \\
\hline 23 & CAK 23A X R- 2000-17-2 & 1.03 & 1.81 & $2.60 *$ & $5.91 * *$ & 2.07 & $-9.48 * *$ & -3.62 & -1.34 \\
\hline 24 & CAK 23A X R-2000-26 & $14.29 * *$ & $7.49 * *$ & $8.33 * *$ & $11.83 * *$ & -6.58 & $-12.36 * *$ & -6.68 & -4.47 \\
\hline 25 & CAK 23A X R-1044-13 & $-7.12 * *$ & $-5.68 * *$ & $-4.95 * *$ & -1.88 & 2.57 & $-14.66 * *$ & $-9.13 *$ & -6.98 \\
\hline 26 & CAK 23A X R-2000-21 & 2.43 & -2.07 & -1.3 & 1.88 & 2.42 & $-14.78 * *$ & $-9.27 *$ & -7.12 \\
\hline 27 & CAK 23A X R-23 & $8.47 * *$ & $2.58 *$ & $3.39 * *$ & $6.72 * *$ & -0.99 & -3.74 & 2.50 & 4.93 \\
\hline 28 & CAK 23A X R-53 & $7.55 * *$ & $3.10 *$ & $3.91 * *$ & $7.26 * *$ & 1.60 & $-7.57 *$ & -1.59 & 0.74 \\
\hline 29 & CAK 23A X AKH-07R & -1.03 & -0.26 & 0.52 & $3.76 * *$ & $16.73 * *$ & -2.87 & 3.41 & 5.86 \\
\hline 30 & CAK 23A X DHY-286-1R & $-6.95 * *$ & $-3.10 *$ & -2.34 & 0.81 & $14.14 * *$ & -5.03 & 1.12 & 3.52 \\
\hline
\end{tabular}


Table.1 Continue...

\begin{tabular}{|c|c|c|c|c|c|c|c|c|c|}
\hline \multirow{2}{*}{$\begin{array}{l}\text { Sr. } \\
\text { No. }\end{array}$} & \multirow[t]{2}{*}{ Crosses } & \multicolumn{4}{|c|}{ Days to $50 \%$ flowering } & \multicolumn{4}{|c|}{ Plant height (cm) } \\
\hline & & ВPH & SH 1 & SH2 & SH3 & ВPH & SH 1 & SH2 & SH3 \\
\hline 31 & CAK 23B X R-42-8 & $-6.31 * *$ & $-4.13 * *$ & $-3.39 * *$ & -0.27 & 3.07 & $-14.24 * *$ & $-8.69 *$ & -6.52 \\
\hline 32 & CAK 23B X R-2000-23 & $-3.73 * *$ & 0.00 & 0.78 & $4.03 * *$ & $10.37 * *$ & 4.17 & $10.91 * *$ & $13.54 * *$ \\
\hline 33 & CAK 23B X R- 2000-17-2 & -1.79 & -1.03 & -0.26 & $2.96 *$ & $11.33 * *$ & -1.28 & 5.11 & $7.60 *$ \\
\hline 34 & CAK 23B X R-2000-26 & 1.65 & $-4.39 * *$ & $-3.65 * *$ & -0.54 & $7.81 *$ & 1.15 & $7.70 *$ & $10.25 * *$ \\
\hline 35 & CAK 23B X R-1044-13 & 0.76 & 2.33 & $3.13 *$ & $6.45 * *$ & $20.03 * *$ & -0.13 & 6.33 & $8.86 *$ \\
\hline 36 & CAK 23B X R-2000-21 & -0.27 & $-4.65 * *$ & $-3.91 * *$ & -0.81 & $14.87 * *$ & -4.43 & 1.76 & 4.17 \\
\hline 37 & CAK 23B X R-23 & $8.47 * *$ & $2.58 *$ & $3.39 * *$ & $6.72 * *$ & $-8.64 *$ & $-11.18 * *$ & -5.43 & -3.19 \\
\hline 38 & CAK 23B X R-53 & $5.12 * *$ & 0.78 & 1.56 & $4.84 * *$ & -5.50 & $-14.02 * *$ & $-8.46 *$ & -6.29 \\
\hline 39 & CAK 23B X AKH-07R & 0.26 & 1.03 & 1.82 & $5.11 * *$ & $9.20 *$ & $-9.14 * *$ & -3.26 & -0.96 \\
\hline 40 & CAK 23B X DHY-286-1R & $-7.44 * *$ & $-3.62 * *$ & $-2.86 *$ & 0.27 & $14.51 * *$ & -4.73 & 1.44 & 3.84 \\
\hline 41 & SRT-1A X R-42-8 & $7.32 * *$ & $9.82 * *$ & $10.68 * *$ & $14.25 * *$ & $-10.72 * *$ & $-21.47 * *$ & $-16.38 * *$ & $-14.40 * *$ \\
\hline 42 & SRT-1A X R-2000-23 & $5.72 * *$ & $9.82 * *$ & $10.68 * *$ & $14.25 * *$ & $-14.54 * *$ & $-19.34 * *$ & $-14.12 * *$ & $-12.08 * *$ \\
\hline 43 & SRT-1A X R- 2000-17-2 & $6.67 * *$ & $7.49 * *$ & $8.33 * *$ & $11.83 * *$ & -2.71 & $-13.72 * *$ & $-8.14 *$ & -5.96 \\
\hline 44 & SRT-1A X R-2000-26 & $4.15 * *$ & $3.88 * *$ & $4.69 * *$ & $8.06 * *$ & -0.60 & $-6.74 *$ & -0.70 & 1.65 \\
\hline 45 & SRT-1A X R-1044-13 & $-5.34 * *$ & $-3.88 * *$ & $-3.13 *$ & 0.00 & -3.87 & $-15.45 * *$ & $-9.97 * *$ & $-7.84 *$ \\
\hline 46 & SRT-1A X R-2000-21 & $-3.63 * *$ & $-3.88 * *$ & $-3.13 *$ & 0.00 & 6.56 & -6.26 & -0.20 & 2.17 \\
\hline 47 & SRT-1A X R-23 & -2.33 & $-2.58 *$ & -1.82 & 1.34 & $7.18 *$ & 4.21 & $10.95 * *$ & $13.59 * *$ \\
\hline 48 & SRT-1A X R-53 & 1.55 & 1.29 & 2.08 & $5.38 * *$ & $8.23 *$ & -1.54 & 4.84 & $7.32 *$ \\
\hline 49 & SRT-1A X AKH-07R & $3.08 *$ & $3.88 * *$ & $4.69 * *$ & $8.06 * *$ & $14.76 * *$ & 0.95 & $7.48 *$ & $10.03 * *$ \\
\hline 50 & SRT-1A X DHY-286-1R & -0.74 & $3.36 * *$ & $4.17 * *$ & $7.53 * *$ & $9.11 *$ & -4.02 & 2.19 & 4.61 \\
\hline 51 & SRT-1B X R-42-8 & $-6.06 * *$ & $-3.88 * *$ & $-3.13 *$ & 0.00 & $23.35 * *$ & $8.51 *$ & $15.53 * *$ & $18.27 * *$ \\
\hline 52 & SRT-1B X R-2000-23 & $-6.47 * *$ & $-2.84 *$ & -2.08 & 1.08 & $9.91 * *$ & 3.74 & $10.45 * *$ & $13.07 * *$ \\
\hline 53 & SRT-1B X R- 2000-17-2 & $-3.08 *$ & -2.33 & -1.56 & 1.61 & 6.61 & -5.46 & 0.66 & 3.05 \\
\hline 54 & SRT-1B X R-2000-26 & $4.15 * *$ & $3.88 * *$ & $4.69 * *$ & $8.06 * *$ & $10.11 * *$ & 3.30 & $9.99 * *$ & $12.60 * *$ \\
\hline 55 & SRT-1B X R-1044-13 & 0.76 & 2.33 & $3.13 *$ & $6.45 * *$ & -1.00 & $-12.92 * *$ & $-7.28 *$ & -5.08 \\
\hline 56 & SRT-1B X R-2000-21 & 1.30 & 1.03 & 1.82 & $5.11 * *$ & $10.11 * *$ & -3.15 & 3.12 & 5.57 \\
\hline 57 & SRT-1B X R-23 & $4.92 * *$ & $4.65 * *$ & $5.47 * *$ & $8.87 * *$ & 3.15 & 0.29 & 6.78 & $9.31 *$ \\
\hline 58 & SRT-1B X R-53 & 2.33 & 2.07 & $2.86 *$ & $6.18 * *$ & -1.12 & $-10.04 * *$ & -4.22 & -1.95 \\
\hline 59 & SRT-1B X AKH-07R & -1.54 & -0.78 & 0.00 & $3.23 *$ & 0.14 & $-11.91 * *$ & -6.21 & -3.99 \\
\hline \multirow[t]{3}{*}{60} & SRT-1B X DHY-286-1R & $-9.43 * *$ & $-5.68 * *$ & $-4.95 * *$ & -1.88 & 4.10 & $-8.43 *$ & -2.51 & -0.20 \\
\hline & S.E. \pm & 0.77 & 0.77 & 0.77 & 0.77 & 3.94 & 3.94 & 3.94 & 3.94 \\
\hline & C.D.@ $9 \%$ & 1.52 & 1.52 & 1.52 & 1.52 & 7.77 & 7.77 & 7.77 & 7.77 \\
\hline
\end{tabular}


Table.1 Continue...

\begin{tabular}{|c|c|c|c|c|c|c|c|c|c|}
\hline \multirow{2}{*}{$\begin{array}{l}\text { Sr. } \\
\text { No. }\end{array}$} & \multirow[t]{2}{*}{ Crosses } & \multicolumn{4}{|c|}{ No. of monopodia per plant } & \multicolumn{4}{|c|}{ Days to $50 \%$ boll burst } \\
\hline & & BPH & SH 1 & SH2 & SH3 & ВPH & SH 1 & SH2 & SH3 \\
\hline 1 & CAK 53A X R-42-8 & -9.51 & -19.93 & $-24.65 *$ & $-35.91 * *$ & 0.86 & $2.63 * *$ & 0.14 & -0.28 \\
\hline 2 & CAK 53A X R-2000-23 & -20.27 & -20.15 & $-24.86 *$ & $-36.09 * *$ & $-4.67 * *$ & $-1.75 *$ & $-4.13 * *$ & $-4.54 * *$ \\
\hline 3 & CAK 53A X R- 2000-17-2 & $-30.11 *$ & $-36.46 * *$ & $-40.21 * *$ & $-49.14 * *$ & $-3.44 * *$ & $-1.75 *$ & $-4.13 * *$ & $-4.54 * *$ \\
\hline 4 & CAK 53A X R-2000-26 & $-32.24 * *$ & $-30.04 *$ & $-34.17 * *$ & $-44.00 * *$ & $-3.87 * *$ & $-2.19 * *$ & $-4.56 * *$ & $-4.96 * *$ \\
\hline 5 & CAK 53A X R-1044-13 & -9.17 & -19.63 & $-24.38 *$ & $-35.68 * *$ & $-3.52 * *$ & 0.00 & $-2.42 * *$ & $-2.84 * *$ \\
\hline 6 & CAK 53A X R-2000-21 & -1.13 & -9.37 & -14.72 & $-27.47 * *$ & $-1.87 * *$ & -0.15 & $-2.56 * *$ & $-2.98 * *$ \\
\hline 7 & CAK 53A X R-23 & 14.8 & 9.89 & 3.40 & -12.05 & $-1.72 *$ & 0.00 & $-2.42 * *$ & $-2.84 * *$ \\
\hline 8 & CAK 53A X R-53 & 0.92 & -10.7 & -15.97 & $-28.53 * *$ & $-1.72 *$ & 0.00 & $-2.42 * *$ & $-2.84 * *$ \\
\hline 9 & CAK 53A X AKH-07R & -4.5 & -15.5 & -20.49 & $-32.37 * *$ & $-4.39 * *$ & $-1.46 *$ & $-3.85 * *$ & $-4.26 * *$ \\
\hline 10 & CAK 53A X DHY-286-1R & 1.89 & 7.45 & 1.11 & -14.00 & $-5.52 * *$ & 0.00 & $-2.42 * *$ & $-2.84 * *$ \\
\hline 11 & CAK 53B X R-42-8 & 10.09 & -2.58 & -8.33 & $-22.03 *$ & -1 & 0.73 & $-1.71 *$ & $-2.13 * *$ \\
\hline 12 & CAK 53B X R-2000-23 & -5.38 & -5.24 & -10.83 & $-24.16 * *$ & $-1.70 *$ & 1.31 & -1.14 & $-1.56 *$ \\
\hline 13 & CAK 53B X R- 2000-17-2 & -12.18 & -20.15 & $-24.86 *$ & $-36.09 * *$ & $-4.02 * *$ & $-2.34 * *$ & $-4.70 * *$ & $-5.11 * *$ \\
\hline 14 & CAK 53B X R-2000-26 & -6.00 & -2.95 & -8.68 & $-22.33 *$ & $-1.72 *$ & 0.00 & $-2.42 * *$ & $-2.84 * *$ \\
\hline 15 & CAK 53B X R-1044-13 & 11.59 & -1.25 & -7.08 & $-20.97 *$ & 0.56 & $4.23 * *$ & $1.71 *$ & 1.28 \\
\hline 16 & CAK 53B X R-2000-21 & 7.09 & -1.85 & -7.64 & $-21.44 *$ & $-2.44 * *$ & -0.73 & $-3.13 * *$ & $-3.55 * *$ \\
\hline 17 & CAK 53B X R-23 & $-29.07 *$ & $-32.10 * *$ & $-36.11 * *$ & $-45.66 * *$ & $-2.73 * *$ & -1.02 & $-3.42 * *$ & $-3.83 * *$ \\
\hline 18 & CAK 53B X R-53 & -1.75 & -13.06 & -18.19 & $-30.42 * *$ & $-2.58 * *$ & -0.88 & $-3.28 * *$ & $-3.69 * *$ \\
\hline 19 & CAK 53B X AKH-07R & -20.35 & $-29.52 *$ & $-33.68 * *$ & $-43.59 * *$ & $-1.42 *$ & $1.61 *$ & -0.85 & -1.28 \\
\hline 20 & CAK 53B X DHY-286-1R & -4.27 & 0.96 & -5.00 & $-19.20 *$ & $-5.93 * *$ & -0.44 & $-2.85 * *$ & $-3.26 * *$ \\
\hline 21 & CAK 23A X R-42-8 & -15.81 & -21.77 & $-26.39 *$ & $-37.39 * *$ & $-3.75 * *$ & $-2.63 * *$ & $-4.99 * *$ & $-5.39 * *$ \\
\hline 22 & CAK 23A X R-2000-23 & -22.62 & -22.51 & $-27.08 *$ & $-37.98 * *$ & $-3.40 * *$ & -0.44 & $-2.85 * *$ & $-3.26 * *$ \\
\hline 23 & CAK 23A X R- 2000-17-2 & -4.92 & -11.66 & -16.88 & $-29.30 * *$ & $2.60 * *$ & $3.80 * *$ & 1.28 & 0.85 \\
\hline 24 & CAK 23A X R-2000-26 & $-37.81 * *$ & $-35.79 * *$ & $-39.58 * *$ & $-48.61 * *$ & $2.61 * *$ & $3.36 * *$ & 0.85 & 0.43 \\
\hline 25 & CAK 23A X R-1044-13 & 7.31 & -0.30 & -6.18 & $-20.20 *$ & $-3.66 * *$ & -0.15 & $-2.56 * *$ & $-2.98 * *$ \\
\hline 26 & CAK 23A X R-2000-21 & $-35.19 * *$ & $-39.78 * *$ & $-43.33 * *$ & $-51.80 * *$ & $1.59 *$ & $2.34 * *$ & -0.14 & -0.57 \\
\hline 27 & CAK 23A X R-23 & $-26.29 *$ & $-29.45 *$ & $-33.61 * *$ & $-43.53 * *$ & $1.73 *$ & $3.07 * *$ & 0.57 & 0.14 \\
\hline 28 & CAK 23A X R-53 & -14.61 & -20.66 & $-25.35 *$ & $-36.50 * *$ & 0.58 & $1.61 *$ & -0.85 & -1.28 \\
\hline 29 & CAK 23A X AKH-07R & 20.89 & 12.32 & 5.69 & -10.1 & -0.99 & $2.04 * *$ & -0.43 & -0.85 \\
\hline 30 & CAK 23A X DHY-286-1R & $-34.99 * *$ & $-31.44 * *$ & $-35.49 * *$ & $-45.13 * *$ & $-3.86 * *$ & $1.75 *$ & -0.71 & -1.13 \\
\hline 31 & CAK 23B X R-42-8 & 9.69 & 1.92 & -4.1 & $-18.43 *$ & -1.3 & -0.15 & $-2.56 * *$ & $-2.98 * *$ \\
\hline
\end{tabular}


Table.1 Continue...

\begin{tabular}{|c|c|c|c|c|c|c|c|c|c|}
\hline \multirow{2}{*}{$\begin{array}{l}\text { Sr. } \\
\text { No. }\end{array}$} & \multirow[t]{2}{*}{ Crosses } & \multicolumn{4}{|c|}{ No. of monopodia per plant } & \multicolumn{4}{|c|}{ Days to $50 \%$ boll burst } \\
\hline & & ВPH & SH 1 & SH2 & SH3 & ВPH & SH 1 & SH2 & SH3 \\
\hline 32 & CAK 23B X R-2000-23 & -14.81 & -14.69 & -19.72 & $-31.72 * *$ & $-1.42 *$ & $1.61 *$ & -0.85 & -1.28 \\
\hline 33 & CAK 23B X R- 2000-17-2 & 0.08 & -7.01 & -12.5 & $-25.58 * *$ & $1.44 *$ & $2.63 * *$ & 0.14 & -0.28 \\
\hline 34 & CAK 23B X R-2000-26 & $-25.88 *$ & $-23.47 *$ & $-27.99 *$ & $-38.75 * *$ & 0.29 & 1.02 & $-1.42 *$ & $-1.84 * *$ \\
\hline 35 & CAK 23B X R-1044-13 & -9.29 & -15.72 & -20.69 & $-32.55 * *$ & $-4.08 * *$ & -0.58 & $-2.99 * *$ & $-3.40 * *$ \\
\hline 36 & CAK 23B X R-2000-21 & -14.38 & -20.44 & $-25.14 *$ & $-36.33 * *$ & $-2.61 * *$ & $-1.90 * *$ & $-4.27 * *$ & $-4.68 * *$ \\
\hline 37 & CAK 23B X R-23 & -11.26 & -15.06 & -20.07 & $-32.01 * *$ & 1.15 & $2.48 * *$ & 0.00 & -0.43 \\
\hline 38 & CAK 23B X R-53 & -17.39 & $-23.25 *$ & $-27.78 *$ & $-38.57 * *$ & 1.16 & $2.19 * *$ & -0.28 & -0.71 \\
\hline 39 & CAK 23B X AKH-07R & -15.81 & -21.77 & $-26.39 *$ & $-37.39 * *$ & $-2.69 * *$ & 0.29 & $-2.14 * *$ & $-2.55 * *$ \\
\hline 40 & CAK 23B X DHY-286-1R & -0.63 & 4.80 & -1.39 & -16.13 & $-3.72 * *$ & $1.90 * *$ & -0.57 & -0.99 \\
\hline 41 & SRT-1A X R-42-8 & -5.75 & -16.53 & -21.46 & $-33.20 * *$ & $4.18 * *$ & $5.40 * *$ & $2.85 * *$ & $2.41 * *$ \\
\hline 42 & SRT-1A X R-2000-23 & -21.15 & -21.03 & $-25.69 *$ & $-36.80 * *$ & $1.42 *$ & $4.53 * *$ & $1.99 * *$ & $1.56 *$ \\
\hline 43 & SRT-1A X R- 2000-17-2 & -12.66 & -20.59 & $-25.28 *$ & $-36.44 * *$ & $2.31 * *$ & $3.50 * *$ & 1.00 & 0.57 \\
\hline 44 & SRT-1A X R-2000-26 & -16.44 & -13.73 & -18.82 & $-30.95 * *$ & $3.48 * *$ & $4.23 * *$ & $1.71 *$ & 1.28 \\
\hline 45 & SRT-1A X R-1044-13 & -3.75 & -14.76 & -19.79 & $-31.78 * *$ & $-4.51 * *$ & -1.02 & $-3.42 * *$ & $-3.83 * *$ \\
\hline 46 & SRT-1A X R-2000-21 & 6.52 & -2.36 & -8.13 & $-21.85 *$ & $-2.32 * *$ & $-1.61 *$ & $-3.99 * *$ & $-4.40 * *$ \\
\hline 47 & SRT-1A X R-23 & -21.59 & $-24.94 *$ & $-29.38 * *$ & $-39.93 * *$ & $1.59 *$ & $2.92 * *$ & 0.43 & 0 \\
\hline 48 & SRT-1A X R-53 & -25.58 & $-34.10 * *$ & $-37.99 * *$ & $-47.25 * *$ & $2.02 * *$ & $3.07 * *$ & 0.57 & 0.14 \\
\hline 49 & SRT-1A X AKH-07R & -22 & $-30.92 * *$ & $-35.00 * *$ & $-44.71 * *$ & 0.42 & $3.50 * *$ & 1.00 & 0.57 \\
\hline 50 & SRT-1A X DHY-286-1R & -10.57 & -5.68 & -11.25 & $-24.51 * *$ & $-6.34 * *$ & -0.88 & $-3.28 * *$ & $-3.69 * *$ \\
\hline 51 & SRT-1B X R-42-8 & -14.75 & $-24.50 *$ & $-28.96 * *$ & $-39.57 * *$ & -0.14 & 1.02 & $-1.42 *$ & $-1.84 * *$ \\
\hline 52 & SRT-1B X R-2000-23 & -12.23 & -12.1 & -17.29 & $-29.65 * *$ & $-3.68 * *$ & -0.73 & $-3.13 * *$ & $-3.55 * *$ \\
\hline 53 & SRT-1B X R- 2000-17-2 & -14.77 & -22.51 & $-27.08 *$ & $-37.98 * *$ & $1.73 *$ & $2.92 * *$ & 0.43 & 0 \\
\hline 54 & SRT-1B X R-2000-26 & -13.72 & -10.92 & -16.18 & $-28.71 * *$ & $3.77 * *$ & $4.53 * *$ & $1.99 * *$ & $1.56 *$ \\
\hline 55 & SRT-1B X R-1044-13 & -11.42 & -21.55 & $-26.18 *$ & $-37.21 * *$ & 0.14 & $3.80 * *$ & 1.28 & 0.85 \\
\hline 56 & SRT-1B X R-2000-21 & -16.59 & $-23.54 *$ & $-28.06 *$ & $-38.81 * *$ & $2.61 * *$ & $3.36 * *$ & 0.85 & 0.43 \\
\hline 57 & SRT-1B X R-23 & -18.97 & -22.44 & $-27.01 *$ & $-37.92 * *$ & $2.02 * *$ & $3.36 * *$ & 0.85 & 0.43 \\
\hline 58 & SRT-1B X R-53 & -1.17 & -12.47 & -17.64 & $-29.95 * *$ & 0.14 & 1.17 & -1.28 & $-1.70 *$ \\
\hline 59 & SRT-1B X AKH-07R & -20.92 & $-29.96 *$ & $-34.10 * *$ & $-43.95 * *$ & -0.28 & $2.77 * *$ & 0.28 & -0.14 \\
\hline \multirow[t]{3}{*}{60} & SRT-1B X DHY-286-1R & $-34.50 * *$ & $-30.92 * *$ & $-35.00 * *$ & $-44.71 * *$ & $-6.76 * *$ & -1.31 & $-3.70 * *$ & $-4.11 * *$ \\
\hline & S.E. \pm & 0.26 & 0.26 & 0.26 & 0.26 & 0.79 & 0.79 & 0.79 & 0.79 \\
\hline & C.D. @ 5\% & 0.52 & 0.52 & 0.52 & 0.52 & 1.56 & 1.56 & 1.56 & 1.56 \\
\hline
\end{tabular}


Table.1 Continue...

\begin{tabular}{|c|c|c|c|c|c|c|c|c|c|}
\hline \multirow{2}{*}{$\begin{array}{l}\text { Sr. } \\
\text { No. }\end{array}$} & \multirow[t]{2}{*}{ Crosses } & \multicolumn{4}{|c|}{ Earliness index } & \multicolumn{4}{|c|}{ No. of sympodia per plant } \\
\hline & & BPH & SH 1 & SH 2 & SH 3 & ВPH & SH 1 & SH 2 & SH 3 \\
\hline 1 & CAK 53A X R-42-8 & $7.24 *$ & -3.15 & 1.78 & -1.72 & 14.43 & 2.15 & -2.18 & -2.3 \\
\hline 2 & CAK 53A X R-2000-23 & 1.00 & -1.94 & 3.05 & -0.49 & 1.42 & -9.47 & -13.31 & -13.41 \\
\hline 3 & CAK 53A X R- 2000-17-2 & -0.5 & -3.39 & 1.53 & -1.97 & -3.26 & -9.47 & -13.31 & -13.41 \\
\hline 4 & CAK 53A X R-2000-26 & $6.89 *$ & $-6.05 *$ & -1.27 & -4.67 & 11.49 & -0.48 & -4.7 & -4.81 \\
\hline 5 & CAK 53A X R-1044-13 & 2.39 & $-6.54 *$ & -1.78 & -5.16 & 11.56 & -0.42 & -4.64 & -4.75 \\
\hline 6 & CAK 53A X R-2000-21 & 5.22 & -2.42 & 2.54 & -0.98 & 11.56 & -0.42 & -4.64 & -4.75 \\
\hline 7 & CAK 53A X R-23 & -2.47 & -4.36 & 0.51 & -2.95 & -2.04 & -6.68 & -10.64 & -10.74 \\
\hline 8 & CAK 53A X R-53 & $-9.52 * *$ & $-7.99 * *$ & -3.31 & $-6.63 *$ & -1.53 & -8.79 & -12.65 & -12.76 \\
\hline 9 & CAK 53A X AKH-07R & -1.18 & 1.21 & $6.36 *$ & 2.7 & $46.85 * *$ & $31.08 * *$ & $25.53 * *$ & $25.38 * *$ \\
\hline 10 & CAK 53A X DHY-286-1R & -4.65 & $-10.65 * *$ & $-6.11 *$ & $-9.34 * *$ & 15.57 & 3.16 & -1.21 & -1.33 \\
\hline 11 & CAK 53B X R-42-8 & -0.54 & $-10.17 * *$ & $-5.60 *$ & $-8.85 * *$ & 2.71 & -8.32 & -12.2 & -12.3 \\
\hline 12 & CAK 53B X R-2000-23 & 4.99 & 1.94 & $7.12 *$ & 3.44 & 16.99 & 4.43 & 0.01 & -0.11 \\
\hline 13 & CAK 53B X R- 2000-17-2 & 4.74 & 1.69 & $6.87 *$ & 3.19 & -2.81 & -9.05 & -12.9 & -13.01 \\
\hline 14 & CAK 53B X R-2000-26 & $6.61 *$ & $-6.30 *$ & -1.53 & -4.91 & 17.33 & 4.74 & 0.30 & 0.18 \\
\hline 15 & CAK 53B X R-1044-13 & 2.12 & $-6.78 *$ & -2.04 & $-5.41 *$ & 6.96 & -4.53 & -8.57 & -8.68 \\
\hline 16 & CAK 53B X R-2000-21 & $8.09 * *$ & 0.24 & 5.34 & 1.72 & 15.92 & 3.47 & -0.91 & -1.03 \\
\hline 17 & CAK 53B X R-23 & -3.46 & $-5.33 *$ & -0.51 & -3.93 & -1.1 & -5.79 & -9.78 & -9.89 \\
\hline 18 & CAK 53B X R-53 & $-10.48 * *$ & $-8.96 * *$ & -4.33 & $-7.62 * *$ & -7.73 & -14.53 & $-18.15 *$ & $-18.24 *$ \\
\hline 19 & CAK 53B X AKH-07R & -2.84 & -0.48 & 4.58 & 0.98 & 9.43 & -2.32 & -6.45 & -6.56 \\
\hline 20 & CAK 53B X DHY-286-1R & -4.91 & $-10.90 * *$ & $-6.36 *$ & $-9.58 * *$ & 5.42 & -5.89 & -9.88 & -9.99 \\
\hline 21 & CAK 23A X R-42-8 & $19.05 * *$ & $8.96 * *$ & $14.50 * *$ & $10.57 * *$ & 2.06 & -6.11 & -10.08 & -10.19 \\
\hline 22 & CAK 23A X R-2000-23 & -4.99 & $-7.75 * *$ & -3.05 & $-6.39 *$ & 14.99 & 5.79 & 1.31 & 1.19 \\
\hline 23 & CAK 23A X R- 2000-17-2 & $-6.23 *$ & $-8.96 * *$ & -4.33 & $-7.62 * *$ & 1.66 & -4.86 & -8.89 & 1.74 \\
\hline 24 & CAK 23A X R-2000-26 & 1.06 & $-7.51 * *$ & -2.8 & $-6.14 *$ & 2.63 & -5.58 & -9.58 & -9.69 \\
\hline 25 & CAK 23A X R-1044-13 & $5.82 *$ & -3.15 & 1.78 & -1.72 & 11.76 & 2.82 & -1.53 & -1.65 \\
\hline 26 & CAK 23A X R-2000-21 & -0.78 & $-7.99 * *$ & -3.31 & $-6.63 *$ & 9.73 & 0.95 & -3.33 & -3.44 \\
\hline 27 & CAK 23A X R-23 & 3.46 & 1.45 & $6.62 *$ & 2.95 & -6.36 & -10.8 & -14.58 & -14.68 \\
\hline 28 & CAK 23A X R-53 & $-5.95 *$ & -4.36 & 0.51 & -2.95 & 3 & -4.59 & -8.63 & -8.74 \\
\hline 29 & CAK 23A X AKH-07R & $-12.29 * *$ & $-10.17 * *$ & $-5.60 *$ & $-8.85 * *$ & -2.86 & -10.63 & -14.42 & -14.52 \\
\hline 30 & CAK 23A X DHY-286-1R & $12.92 * *$ & $5.81 *$ & $11.20 * *$ & $7.37 * *$ & -3.99 & -11.67 & -15.41 & -15.52 \\
\hline 31 & CAK 23B X R-42-8 & 1.59 & $-7.02 * *$ & -2.29 & $-5.65 *$ & $-19.68 *$ & $-26.11 * *$ & $-29.23 * *$ & $-29.32 * *$ \\
\hline
\end{tabular}


Table.1 Continue...

\begin{tabular}{|c|c|c|c|c|c|c|c|c|c|}
\hline \multirow{2}{*}{$\begin{array}{l}\text { Sr. } \\
\text { No. }\end{array}$} & \multirow[t]{2}{*}{ Crosses } & \multicolumn{4}{|c|}{ Earliness index } & \multicolumn{4}{|c|}{ No. of sympodia per plant } \\
\hline & & ВPH & SH 1 & SH 2 & SH 3 & ВPH & SH 1 & SH 2 & SH 3 \\
\hline 32 & CAK 23B X R-2000-23 & -2.99 & $-5.81 *$ & -1.02 & -4.42 & $29.66 * *$ & $19.28 *$ & 14.23 & 14.1 \\
\hline 33 & CAK 23B X R- 2000-17-2 & $-6.48 *$ & $-9.20 * *$ & -4.58 & $-7.86 * *$ & 1.72 & -4.81 & -8.84 & -8.95 \\
\hline 34 & CAK 23B X R-2000-26 & 0.00 & $-8.47 * *$ & -3.82 & $-7.13 * *$ & -4.79 & -12.41 & -16.12 & -16.22 \\
\hline 35 & CAK 23B X R-1044-13 & $11.64 * *$ & 2.18 & $7.38 * *$ & 3.69 & 4.03 & -4.29 & -8.35 & -8.46 \\
\hline 36 & CAK 23B X R-2000-21 & $7.31 *$ & -0.48 & 4.58 & 0.98 & 14.99 & 5.79 & 1.31 & 1.19 \\
\hline 37 & CAK 23B X R-23 & $9.63 * *$ & $7.51 * *$ & $12.98 * *$ & $9.09 * *$ & 4.33 & -0.61 & -4.82 & -4.93 \\
\hline 38 & CAK 23B X R-53 & -2.62 & -0.97 & 4.07 & 0.49 & 4.15 & -3.53 & -7.61 & -7.72 \\
\hline 39 & CAK 23B X AKH-07R & -0.71 & 1.69 & $6.87 *$ & 3.19 & $34.20 * *$ & $23.46 * *$ & $18.24 *$ & $18.09 *$ \\
\hline 40 & CAK 23B X DHY-286-1R & $17.83 * *$ & $10.41 * *$ & $16.03 * *$ & $12.04 * *$ & $25.64 * *$ & 15.59 & 10.7 & 10.56 \\
\hline 41 & SRT-1A X R-42-8 & -1.53 & $-6.30 *$ & -1.53 & -4.91 & -13.9 & $-28.54 * *$ & $-31.56 * *$ & $-31.65 * *$ \\
\hline 42 & SRT-1A X R-2000-23 & 1.5 & -1.45 & 3.56 & 0.00 & -2.92 & -16.00 & $-19.56 *$ & $-19.65 *$ \\
\hline 43 & SRT-1A X R- 2000-17-2 & 4.74 & 1.69 & $6.87 *$ & 3.19 & -9.45 & -15.26 & $-18.85 *$ & $-18.95 *$ \\
\hline 44 & SRT-1A X R-2000-26 & $-5.85 *$ & $-10.41 * *$ & $-5.85 *$ & $-9.09 * *$ & $22.00 *$ & 3.51 & -0.88 & -1.00 \\
\hline 45 & SRT-1A X R-1044-13 & $15.52 * *$ & $9.93 * *$ & $15.52 * *$ & $11.55 * *$ & 13.68 & -11.72 & -15.45 & -15.56 \\
\hline 46 & SRT-1A X R-2000-21 & 1.02 & -3.87 & 1.02 & -2.46 & 7.22 & -9.68 & -13.51 & -13.61 \\
\hline 47 & SRT-1A X R-23 & 2.72 & 0.73 & $5.85 *$ & 2.21 & -6.85 & -11.26 & -15.02 & -15.12 \\
\hline 48 & SRT-1A X R-53 & $-9.95 * *$ & $-8.43 * *$ & -3.77 & $-7.08 *$ & -7.16 & -14.00 & $-17.64 *$ & $-17.74 *$ \\
\hline 49 & SRT-1A X AKH-07R & 1.42 & 3.87 & $9.16 * *$ & $5.41 *$ & 12.2 & -14.44 & $-18.06 *$ & $-18.16 *$ \\
\hline 50 & SRT-1A X DHY-286-1R & $-8.14 * *$ & $-12.59 * *$ & $-8.14 * *$ & $-11.30 * *$ & -4.63 & $-20.79 *$ & $-24.14 * *$ & $-24.23 * *$ \\
\hline 51 & SRT-1B X R-42-8 & -2.29 & $-7.02 * *$ & -2.29 & $-5.65 *$ & -4.25 & $-20.53 *$ & $-23.89 * *$ & $-23.98 * *$ \\
\hline 52 & SRT-1B X R-2000-23 & -5.24 & $-7.99 * *$ & -3.31 & $-6.63 *$ & 5.96 & -8.32 & -12.2 & -12.3 \\
\hline 53 & SRT-1B X R- 2000-17-2 & $10.72 * *$ & $7.51 * *$ & $12.98 * *$ & $9.09 * *$ & -7.96 & -13.87 & $-17.52 *$ & $-17.62 *$ \\
\hline 54 & SRT-1B X R-2000-26 & -4.58 & $-9.20 * *$ & -4.58 & $-7.86 * *$ & -9.43 & $-23.16 * *$ & $-26.41 * *$ & $-26.50 * *$ \\
\hline 55 & SRT-1B X R-1044-13 & 5.34 & 0.24 & 5.34 & 1.72 & 13.31 & -12.00 & -15.73 & -15.83 \\
\hline 56 & SRT-1B X R-2000-21 & $-5.85 *$ & $-10.41 * *$ & $-5.85 *$ & $-9.09 * *$ & -4.52 & $-19.58 *$ & $-22.98 * *$ & $-23.08 * *$ \\
\hline 57 & SRT-1B X R-23 & $7.41 * *$ & $5.33 *$ & $10.69 * *$ & $6.88 *$ & -1.55 & -6.21 & -10.18 & -10.29 \\
\hline 58 & SRT-1B X R-53 & $-8.57 * *$ & $-7.02 * *$ & -2.29 & $-5.65 *$ & 11.14 & 2.95 & -1.41 & -1.53 \\
\hline 59 & SRT-1B X AKH-07R & -1.42 & 0.97 & $6.11 *$ & 2.46 & $33.95 * *$ & 2.14 & -2.19 & -2.31 \\
\hline \multirow[t]{3}{*}{60} & SRT-1B X DHY-286-1R & -3.05 & $-7.75 * *$ & -3.05 & $-6.39 *$ & 16.22 & -3.47 & -7.56 & -7.67 \\
\hline & S.E. \pm & 0.02 & 0.02 & 0.02 & 0.02 & 1.37 & 1.37 & 1.37 & 1.37 \\
\hline & C.D. @ 5\% & 0.04 & 0.04 & 0.04 & 0.04 & 2.70 & 2.70 & 2.70 & 2.70 \\
\hline
\end{tabular}


Table.1 Continue...

\begin{tabular}{|c|c|c|c|c|c|c|c|c|c|}
\hline \multirow{2}{*}{$\begin{array}{l}\text { Sr. } \\
\text { No. }\end{array}$} & \multirow[t]{2}{*}{ Crosses } & \multicolumn{4}{|c|}{ No. of bolls per plant } & \multicolumn{4}{|c|}{ Boll weight (g) } \\
\hline & & ВPH & SH 1 & SH 2 & SH 3 & ВPH & SH 1 & SH 2 & SH 3 \\
\hline 1 & CAK 53A X R-42-8 & 12.22 & -12.3 & -9.14 & -0.37 & -10.84 & -11.41 & $-23.03 *$ & $-26.65 * *$ \\
\hline 2 & CAK 53A X R-2000-23 & 1.52 & -15.05 & -11.98 & -3.49 & -1.68 & 8.22 & -5.98 & -10.4 \\
\hline 3 & CAK 53A X R- 2000-17-2 & -3.56 & -10.52 & -7.29 & 1.66 & 3.01 & 9.11 & -5.2 & -9.66 \\
\hline 4 & CAK 53A X R-2000-26 & -1.26 & -14.39 & -11.31 & -2.75 & 8.87 & 3.25 & -10.3 & -14.51 \\
\hline 5 & CAK 53A X R-1044-13 & 25.02 & -14.61 & -11.53 & -2.99 & -9.8 & -11.98 & $-23.53 *$ & $-27.12 * *$ \\
\hline 6 & CAK 53A X R-2000-21 & 20.55 & -17.66 & -14.69 & -6.46 & 7.00 & 8.16 & -6.04 & -10.45 \\
\hline 7 & CAK 53A X R-23 & -11.05 & $-32.26 * *$ & $-29.81 * *$ & $-23.04 *$ & $-19.67 *$ & -7.84 & $-19.93 *$ & $-23.69 * *$ \\
\hline 8 & CAK 53A X R-53 & 15.31 & $-21.24 *$ & -18.4 & -10.53 & -2.97 & -4.14 & -16.72 & $-20.63 *$ \\
\hline 9 & CAK 53A X AKH-07R & $113.58 * *$ & $56.00 * *$ & $61.63 * *$ & $77.23 * *$ & $43.55 * *$ & $36.14 * *$ & 18.27 & 12.72 \\
\hline 10 & CAK 53A X DHY-286-1R & -1.69 & $-32.85 * *$ & $-30.43 * *$ & $-23.72 *$ & -10.16 & -1.91 & -14.78 & $-18.79 *$ \\
\hline 11 & CAK 53B X R-42-8 & $48.14 * *$ & 15.78 & $19.95 *$ & $31.53 * *$ & 6.03 & 5.35 & -8.47 & -12.77 \\
\hline 12 & CAK 53B X R-2000-23 & 17.47 & -1.7 & 1.84 & 11.67 & 11.00 & $22.18 *$ & 6.15 & 1.16 \\
\hline 13 & CAK 53B X R- 2000-17-2 & $28.91 * *$ & $19.61 *$ & $23.92 *$ & $35.88 * *$ & 14.62 & 21.41 & 5.48 & 0.53 \\
\hline 14 & CAK 53B X R-2000-26 & $48.71 * *$ & $28.92 * *$ & $33.57 * *$ & $46.46 * *$ & 20.23 & 14.02 & -0.94 & -5.59 \\
\hline 15 & CAK 53B X R-1044-13 & $28.61 *$ & -12.16 & -8.99 & -0.21 & -0.07 & -2.49 & -15.28 & $-19.26 *$ \\
\hline 16 & CAK 53B X R-2000-21 & $35.62 *$ & -7.37 & -4.03 & 5.23 & -4.29 & -3.25 & -15.95 & $-19.89 *$ \\
\hline 17 & CAK 53B X R-23 & 20.13 & -8.51 & -5.21 & 3.93 & -6.94 & 6.76 & -7.25 & -11.61 \\
\hline 18 & CAK 53B X R-53 & $43.04 * *$ & -2.3 & 1.22 & 10.99 & $29.48 * *$ & $27.92 *$ & 11.13 & 5.91 \\
\hline 19 & CAK 53B X AKH-07R & $63.27 * *$ & $19.26 *$ & $23.56 *$ & $35.49 * *$ & 1.28 & -3.95 & -16.56 & $-20.47 *$ \\
\hline 20 & CAK 53B X DHY-286-1R & 10.59 & $-24.47 *$ & $-21.74 *$ & -14.19 & -8.70 & -0.32 & -13.4 & -17.47 \\
\hline 21 & CAK 23A X R-42-8 & 13.93 & -10.96 & -7.75 & 1.15 & 5.39 & 4.72 & -9.03 & -13.3 \\
\hline 22 & CAK 23A X R-2000-23 & $23.19 *$ & 3.09 & 6.8 & 17.11 & 2.32 & 12.62 & -2.16 & -6.75 \\
\hline 23 & CAK 23A X R- 2000-17-2 & -1.94 & -9.01 & -5.73 & 3.37 & 13.9 & 20.65 & 4.82 & -0.11 \\
\hline 24 & CAK 23A X R-2000-26 & -10.8 & $-22.67 *$ & $-19.88 *$ & -12.15 & $24.31 *$ & 20.59 & 4.76 & -0.16 \\
\hline 25 & CAK 23A X R-1044-13 & 23.86 & -12 & -8.82 & -0.02 & $27.37 *$ & $24.28 *$ & 7.97 & 2.9 \\
\hline 26 & CAK 23A X R-2000-21 & 7.46 & $-23.65 *$ & $-20.90 *$ & -13.26 & $21.69 *$ & $23.01 *$ & 6.87 & 1.85 \\
\hline 27 & CAK 23A X R-23 & -12.31 & $-33.21 * *$ & $-30.81 * *$ & $-24.13 *$ & 0.39 & 15.17 & 0.06 & -4.64 \\
\hline 28 & CAK 23A X R-53 & 3.14 & $-26.72 * *$ & $-24.07 *$ & -16.75 & 3.03 & 1.78 & -11.57 & -15.73 \\
\hline 29 & CAK 23A X AKH-07R & 13.1 & -17.39 & -14.41 & -6.15 & -3.55 & -6.44 & $-18.72 *$ & $-22.53 *$ \\
\hline 30 & CAK 23A X DHY-286-1R & 23.32 & -12.38 & -9.22 & -0.46 & -13.89 & -5.99 & -18.33 & $-22.16 *$ \\
\hline 31 & CAK 23B X R-42-8 & 3.9 & $-18.80 *$ & -15.88 & -7.76 & $23.41 *$ & $22.63 *$ & 6.53 & 1.53 \\
\hline
\end{tabular}


Table.1 Continue...

\begin{tabular}{|c|c|c|c|c|c|c|c|c|c|}
\hline \multirow{2}{*}{$\begin{array}{l}\text { Sr. } \\
\text { No. }\end{array}$} & \multirow[t]{2}{*}{ Crosses } & \multicolumn{4}{|c|}{ No. of bolls per plant } & \multicolumn{4}{|c|}{ Boll weight (g) } \\
\hline & & ВPH & SH 1 & SH 2 & SH 3 & ВPH & SH 1 & SH 2 & SH 3 \\
\hline 32 & CAK 23B X R-2000-23 & $74.42 * *$ & $45.96 * *$ & $51.22 * *$ & $65.81 * *$ & $23.68 *$ & $36.14 * *$ & 18.27 & 12.72 \\
\hline 33 & CAK 23B X R- 2000-17-2 & -13.23 & $-19.49 *$ & -16.59 & -8.54 & 5.05 & 11.28 & -3.32 & -7.86 \\
\hline 34 & CAK 23B X R-2000-26 & 3.58 & -10.2 & -6.96 & 2.02 & 11.37 & 8.03 & -6.15 & -10.55 \\
\hline 35 & CAK 23B X R-1044-13 & $50.83 * *$ & 7.17 & 11.03 & $21.75 *$ & $24.56 *$ & $21.54 *$ & 5.59 & 0.63 \\
\hline 36 & CAK 23B X R-2000-21 & 21.02 & -14.01 & -10.91 & -2.31 & $24.59 *$ & $25.94 *$ & 9.41 & 4.27 \\
\hline 37 & CAK 23B X R-23 & 9.9 & -16.3 & -13.28 & -4.91 & 8.89 & $24.92 *$ & 8.53 & 3.43 \\
\hline 38 & CAK 23B X R-53 & 17.04 & -16.84 & -13.85 & -5.53 & $22.97 *$ & 21.48 & 5.54 & 0.58 \\
\hline 39 & CAK 23B X AKH-07R & $89.73 * *$ & $38.59 * *$ & $43.58 * *$ & $57.44 * *$ & $52.76 * *$ & $48.18 * *$ & $28.74 * *$ & $22.69 *$ \\
\hline 40 & CAK 23B X DHY-286-1R & $82.29 * *$ & $29.52 * *$ & $34.19 * *$ & $47.14 * *$ & 4.09 & 13.64 & -1.27 & -5.91 \\
\hline 41 & SRT-1A X R-42-8 & -15.89 & $-34.27 * *$ & $-31.90 * *$ & $-25.33 *$ & -16.96 & -7.33 & $-19.49 *$ & $-23.27 *$ \\
\hline 42 & SRT-1A X R-2000-23 & $-34.26 * *$ & $-44.99 * *$ & $-43.00 * *$ & $-37.50 * *$ & 13.08 & $26.20 *$ & 9.63 & 4.49 \\
\hline 43 & SRT-1A X R- 2000-17-2 & -6.98 & -13.7 & -10.58 & -1.95 & 5.31 & 17.53 & 2.1 & -2.69 \\
\hline 44 & SRT-1A X R-2000-26 & $39.96 * *$ & $21.34 *$ & $25.71 * *$ & $37.84 * *$ & 18.85 & $32.63 * *$ & 15.23 & 9.82 \\
\hline 45 & SRT-1A X R-1044-13 & 18.1 & -15.14 & -12.09 & -3.6 & 0.69 & 12.36 & -2.38 & -6.97 \\
\hline 46 & SRT-1A X R-2000-21 & 14.56 & -17.69 & -14.72 & -6.49 & -0.34 & 11.22 & -3.38 & -7.92 \\
\hline 47 & SRT-1A X R-23 & 5.5 & $-19.65 *$ & -16.76 & -8.72 & $-26.50 * *$ & -15.68 & $-26.74 * *$ & $-30.18 * *$ \\
\hline 48 & SRT-1A X R-53 & $38.64 * *$ & -0.39 & 3.2 & 13.17 & -17.13 & -7.52 & $-19.66 *$ & $-23.43 *$ \\
\hline 49 & SRT-1A X AKH-07R & 25.07 & -8.64 & -5.35 & 3.79 & -1.48 & 9.94 & -4.49 & -8.97 \\
\hline 50 & SRT-1A X DHY-286-1R & 19.09 & -14.43 & -11.35 & -2.79 & -10.62 & -0.25 & -13.34 & -17.41 \\
\hline 51 & SRT-1B X R-42-8 & $24.76 *$ & -2.5 & 1.02 & 10.76 & -3.48 & 7.71 & -6.42 & -10.82 \\
\hline 52 & SRT-1B X R-2000-23 & $40.17 * *$ & 17.29 & $21.52 *$ & $33.25 * *$ & 12.74 & $25.81 *$ & 9.3 & 4.17 \\
\hline 53 & SRT-1B X R- 2000-17-2 & 10.82 & 2.82 & 6.53 & 16.81 & 15.31 & $28.68 * *$ & 11.79 & 6.54 \\
\hline 54 & SRT-1B X R-2000-26 & 6.87 & -7.35 & -4.01 & 5.26 & -1.03 & 10.45 & -4.04 & -8.55 \\
\hline 55 & SRT-1B X R-1044-13 & $65.93 * *$ & $19.22 *$ & $23.52 *$ & $35.44 * *$ & 6.74 & 19.12 & 3.49 & -1.37 \\
\hline 56 & SRT-1B X R-2000-21 & 0.76 & $-27.60 * *$ & $-24.99 *$ & -17.76 & 2.28 & 14.15 & -0.83 & -5.49 \\
\hline 57 & SRT-1B X R-23 & 19.59 & -8.93 & -5.64 & 3.46 & -4.78 & 9.24 & -5.09 & -9.55 \\
\hline 58 & SRT-1B X R-53 & $49.64 * *$ & 7.52 & 11.39 & $22.14 *$ & -10.28 & 0.13 & -13.01 & -17.1 \\
\hline 59 & SRT-1B X AKH-07R & -0.84 & $-27.57 * *$ & $-24.96 *$ & -17.72 & -0.57 & 10.96 & -3.6 & -8.13 \\
\hline \multirow[t]{3}{*}{60} & SRT-1B X DHY-286-1R & 18.97 & -14.52 & -11.44 & -2.89 & -16.9 & -7.27 & $-19.44 *$ & $-23.22 *$ \\
\hline & S.E. \pm & 2.91 & 2.91 & 2.91 & 2.91 & 0.28 & 0.28 & 0.28 & 0.28 \\
\hline & C.D. @ 5\% & 5.75 & 5.75 & 5.75 & 5.75 & 0.56 & 0.56 & 0.56 & 0.56 \\
\hline
\end{tabular}


Table.1 Continue...

\begin{tabular}{|c|c|c|c|c|c|c|c|c|c|}
\hline \multirow{2}{*}{$\begin{array}{l}\text { Sr. } \\
\text { No. }\end{array}$} & \multirow[t]{2}{*}{ Crosses } & \multicolumn{4}{|c|}{ Seed cotton yield per plant (gm) } & \multicolumn{4}{|c|}{ Seed index } \\
\hline & & BPH & SH 1 & SH 2 & SH 3 & BPH & SH 1 & SH 2 & SH 3 \\
\hline 1 & CAK 53A X R-42-8 & $-24.76 * *$ & $-47.56 * *$ & $-19.51 * *$ & $-14.79 *$ & $-15.73 * *$ & $-11.08 * *$ & $18.58 * *$ & $31.24 * *$ \\
\hline 2 & CAK 53A X R-2000-23 & -3.83 & $-43.93 * *$ & $-13.94 *$ & -8.89 & $10.75 *$ & 7.42 & $43.26 * *$ & $58.56 * *$ \\
\hline 3 & CAK 53A X R- 2000-17-2 & $-33.55 * *$ & $-45.75 * *$ & $-16.74 * *$ & -11.86 & $-10.95 * *$ & -6.62 & $24.54 * *$ & $37.83 * *$ \\
\hline 4 & CAK 53A X R-2000-26 & $53.69 * *$ & $-10.20 * *$ & $37.82 * *$ & $45.91 * *$ & 3.22 & $9.13 *$ & $45.53 * *$ & $61.07 * *$ \\
\hline 5 & CAK 53A X R-1044-13 & $64.73 * *$ & $-19.03 * *$ & $24.27 * *$ & $31.56 * *$ & 2.94 & 4.45 & $39.29 * *$ & $54.16 * *$ \\
\hline 6 & CAK 53A X R-2000-21 & $32.58 * *$ & $-45.24 * *$ & $-15.95 * *$ & -11.02 & -4.18 & -2.57 & $29.93 * *$ & $43.80 * *$ \\
\hline 7 & CAK 53A X R-23 & $-11.30 *$ & $-40.31 * *$ & -8.39 & -3.02 & 1.21 & 6.79 & $42.41 * *$ & $57.61 * *$ \\
\hline 8 & CAK 53A X R-53 & -7.77 & $-37.44 * *$ & -3.98 & 1.65 & 4.80 & 2.11 & $36.17 * *$ & $50.71 * *$ \\
\hline 9 & CAK 53A X AKH-07R & $195.47 * *$ & $20.70 * *$ & $85.26 * *$ & $96.12 * *$ & $9.87 *$ & 2.32 & $36.45 * *$ & $51.02 * *$ \\
\hline 10 & CAK 53A X DHY-286-1R & 12.70 & $-53.96 * *$ & $-29.34 * *$ & $-25.20 * *$ & -0.20 & -3.85 & $28.23 * *$ & $41.92 * *$ \\
\hline 11 & CAK 53B X R-42-8 & -9.87 & $-37.18 * *$ & -3.58 & 2.07 & $-11.49 * *$ & -6.62 & $24.54 * *$ & $37.83 * *$ \\
\hline 12 & CAK 53B X R-2000-23 & $65.49 * *$ & -3.51 & $48.09 * *$ & $56.78 * *$ & -4.82 & -7.68 & $23.12 * *$ & $36.26 * *$ \\
\hline 13 & CAK 53B X R- 2000-17-2 & $-18.06 * *$ & $-33.10 * *$ & 2.68 & 8.7 & 6.90 & $12.10 * *$ & $49.50 * *$ & $65.46 * *$ \\
\hline 14 & CAK 53B X R-2000-26 & $41.50 * *$ & $-17.32 * *$ & $26.90 * *$ & $34.34 * *$ & 0.40 & 6.15 & $41.56 * *$ & $56.67 * *$ \\
\hline 15 & CAK 53B X R-1044-13 & 3.54 & $-49.11 * *$ & $-21.89 * *$ & $-17.31 * *$ & 6.08 & 7.64 & $43.55 * *$ & $58.87 * *$ \\
\hline 16 & CAK 53B X R-2000-21 & $37.47 * *$ & $-43.22 * *$ & $-12.86 *$ & -7.74 & $11.92 * *$ & $13.81 * *$ & $51.77 * *$ & $67.97 * *$ \\
\hline 17 & CAK 53B X R-23 & $21.61 * *$ & $-18.17 * *$ & $25.60 * *$ & $32.97 * *$ & $-9.07 *$ & -4.06 & $27.94 * *$ & $41.60 * *$ \\
\hline 18 & CAK 53B X R-53 & $24.16 * *$ & $-15.79 * *$ & $29.25 * *$ & $36.84 * *$ & $13.10 * *$ & $10.19 *$ & $46.95 * *$ & $62.64 * *$ \\
\hline 19 & CAK 53B X AKH-07R & $131.51 * *$ & -5.43 & $45.15 * *$ & $53.67 * *$ & $17.86 * *$ & $9.76 *$ & $46.38 * *$ & $62.01 * *$ \\
\hline 20 & CAK 53B X DHY-286-1R & $59.15 * *$ & $-34.99 * *$ & -0.21 & 5.64 & $16.14 * *$ & $11.89 * *$ & $49.22 * *$ & $65.15 * *$ \\
\hline 21 & CAK 23A X R-42-8 & $49.05 * *$ & 3.88 & $59.44 * *$ & $68.80 * *$ & 4.36 & $12.10 * *$ & $49.50 * *$ & $65.46 * *$ \\
\hline 22 & CAK 23A X R-2000-23 & $75.77 * *$ & 2.72 & $57.66 * *$ & $66.91 * *$ & -3.96 & 3.17 & $37.59 * *$ & $52.28 * *$ \\
\hline 23 & CAK 23A X R- 2000-17-2 & $-9.44 *$ & $-26.07 * *$ & $13.47 *$ & $20.13 * *$ & -0.59 & 6.79 & $42.41 * *$ & $57.61 * *$ \\
\hline 24 & CAK 23A X R-2000-26 & $-38.80 * *$ & $-64.23 * *$ & $-45.10 * *$ & $-41.88 * *$ & 2.18 & $9.76 *$ & $46.38 * *$ & $62.01 * *$ \\
\hline 25 & CAK 23A X R-1044-13 & $40.25 * *$ & $-18.04 * *$ & $25.80 * *$ & $33.18 * *$ & 0.99 & $8.49 *$ & $44.68 * *$ & $60.13 * *$ \\
\hline 26 & CAK 23A X R-2000-21 & 11.66 & $-34.74 * *$ & 0.16 & 6.04 & $-7.92 *$ & -1.08 & $31.91 * *$ & $46.00 * *$ \\
\hline 27 & CAK 23A X R-23 & $-37.94 * *$ & $-58.24 * *$ & $-35.91 * *$ & $-32.15 * *$ & -4.55 & 2.53 & $36.74 * *$ & $51.33 * *$ \\
\hline 28 & CAK 23A X R-53 & -3.36 & $-34.45 * *$ & 0.61 & 6.51 & -0.59 & 6.79 & $42.41 * *$ & $57.61 * *$ \\
\hline 29 & CAK 23A X AKH-07R & 4.94 & $-38.67 * *$ & -5.87 & -0.34 & 0.40 & 7.85 & $43.83 * *$ & $59.18 * *$ \\
\hline 30 & CAK 23A X DHY-286-1R & $-32.69 * *$ & $-60.66 * *$ & $-39.63 * *$ & $-36.09 * *$ & 2.38 & $9.98 *$ & $46.67 * *$ & $62.32 * *$ \\
\hline 31 & CAK 23B X R-42-8 & $-32.56 * *$ & $-53.00 * *$ & $-27.86 * *$ & $-23.63 * *$ & 1.98 & $9.55 *$ & $46.10 * *$ & $61.70 * *$ \\
\hline
\end{tabular}


Table.1 Continue...

\begin{tabular}{|c|c|c|c|c|c|c|c|c|c|}
\hline \multirow{2}{*}{$\begin{array}{l}\text { Sr. } \\
\text { No. }\end{array}$} & \multirow[t]{2}{*}{ Crosses } & \multicolumn{4}{|c|}{ Seed cotton yield per plant (g) } & \multicolumn{4}{|c|}{ Seed index $(g)$} \\
\hline & & ВPH & SH 1 & SH 2 & SH 3 & ВPH & SH 1 & SH 2 & SH 3 \\
\hline 32 & CAK 23B X R-2000-23 & $88.85 * *$ & $10.37 * *$ & $69.39 * *$ & $79.33 * *$ & 3.17 & $10.83 * *$ & $47.80 * *$ & $63.58 * *$ \\
\hline 33 & CAK 23B X R- 2000-17-2 & -8.88 & $-25.61 * *$ & $14.18 *$ & $20.87 * *$ & 0.20 & 7.64 & $43.55 * *$ & $58.87 * *$ \\
\hline 34 & CAK 23B X R-2000-26 & $12.75 *$ & $-34.11 * *$ & 1.13 & 7.07 & -5.35 & 1.68 & $35.60 * *$ & $50.08 * *$ \\
\hline 35 & CAK 23B X R-1044-13 & $74.78 * *$ & 2.15 & $56.78 * *$ & $65.98 * *$ & 3.96 & $11.68 * *$ & $48.94 * *$ & $64.84 * *$ \\
\hline 36 & CAK 23B X R-2000-21 & $12.69 *$ & $-34.14 * *$ & 1.09 & 7.02 & $-10.89 * *$ & -4.28 & $27.66 * *$ & $41.29 * *$ \\
\hline 37 & CAK 23B X R-23 & 5.89 & $-28.75 * *$ & 9.35 & $15.77 *$ & -2.18 & 5.08 & $40.14 * *$ & $55.10 * *$ \\
\hline 38 & CAK 23B X R-53 & $24.85 * *$ & $-15.32 * *$ & $29.97 * *$ & $37.60 * *$ & $-10.89 * *$ & -4.28 & $27.66 * *$ & $41.29 * *$ \\
\hline 39 & CAK 23B X AKH-07R & $95.06 * *$ & $14.00 * *$ & $74.97 * *$ & $85.23 * *$ & $-8.91 *$ & -2.15 & $30.50 * *$ & $44.43 * *$ \\
\hline 40 & CAK 23B X DHY-286-1R & $93.64 * *$ & $13.17 * *$ & $73.70 * *$ & $83.89 * *$ & $8.12 *$ & $16.15 * *$ & $54.89 * *$ & $71.43 * *$ \\
\hline 41 & SRT-1A X R-42-8 & $21.29 * *$ & $-15.46 * *$ & $29.75 * *$ & $37.36 * *$ & -1.21 & 4.23 & $39.01 * *$ & $53.85 * *$ \\
\hline 42 & SRT-1A X R-2000-23 & 2.67 & $-40.14 * *$ & -8.13 & -2.74 & 8.11 & 4.87 & $39.86 * *$ & $54.79 * *$ \\
\hline 43 & SRT-1A X R- 2000-17-2 & $-38.88 * *$ & $-50.10 * *$ & $-23.41 * *$ & $-18.92 * *$ & 2.64 & 7.64 & $43.55 * *$ & $58.87 * *$ \\
\hline 44 & SRT-1A X R-2000-26 & $87.97 * *$ & $9.83 * *$ & $68.57 * *$ & $78.46 * *$ & 2.41 & $8.27 *$ & $44.40 * *$ & $59.81 * *$ \\
\hline 45 & SRT-1A X R-1044-13 & $55.13 * *$ & $-23.75 * *$ & $17.03 * *$ & $23.89 * *$ & -4.61 & -3.21 & $29.08 * *$ & $42.86 * *$ \\
\hline 46 & SRT-1A X R-2000-21 & $79.32 * *$ & $-25.94 * *$ & $13.67 *$ & $20.34 * *$ & 2.30 & 4.02 & $38.72 * *$ & $53.53 * *$ \\
\hline 47 & SRT-1A X R-23 & $-42.33 * *$ & $-61.19 * *$ & $-40.44 * *$ & $-36.94 * *$ & 1.81 & 7.42 & $43.26 * *$ & $58.56 * *$ \\
\hline 48 & SRT-1A X R-53 & $-17.87 * *$ & $-44.29 * *$ & $-14.50 *$ & -9.48 & 5.68 & 2.96 & $37.30 * *$ & $51.96 * *$ \\
\hline 49 & SRT-1A X AKH-07R & $171.06 * *$ & 4.22 & $59.96 * *$ & $69.35 * *$ & $19.69 * *$ & $11.47 * *$ & $48.65 * *$ & $64.52 * *$ \\
\hline 50 & SRT-1A X DHY-286-1R & $36.62 * *$ & $-47.47 * *$ & $-19.38 * *$ & $-14.65 *$ & $13.49 * *$ & $9.34 *$ & $45.82 * *$ & $61.38 * *$ \\
\hline 51 & SRT-1B X R-42-8 & $23.03 * *$ & $-14.25 * *$ & $31.61 * *$ & $39.33 * *$ & 4.84 & $10.61 *$ & $47.52 * *$ & $63.27 * *$ \\
\hline 52 & SRT-1B X R-2000-23 & $61.53 * *$ & -5.82 & $44.55 * *$ & $53.03 * *$ & $13.38 * *$ & $9.98 *$ & $46.67 * *$ & $62.32 * *$ \\
\hline 53 & SRT-1B X R- 2000-17-2 & -1.46 & $-19.55 * *$ & $23.48 * *$ & $30.72 * *$ & 1.42 & 6.36 & $41.84 * *$ & $56.99 * *$ \\
\hline 54 & SRT-1B X R-2000-26 & $41.48 * *$ & $-17.33 * *$ & $26.88 * *$ & $34.32 * *$ & 3.42 & $9.34 *$ & $45.82 * *$ & $61.38 * *$ \\
\hline 55 & SRT-1B X R-1044-13 & $28.60 * *$ & $-36.79 * *$ & -2.99 & 2.71 & 1.26 & 2.74 & $37.02 * *$ & $51.65 * *$ \\
\hline 56 & SRT-1B X R-2000-21 & $82.73 * *$ & $-24.53 * *$ & $15.83 * *$ & $22.63 * *$ & 3.14 & 4.87 & $39.86 * *$ & $54.79 * *$ \\
\hline 57 & SRT-1B X R-23 & $19.19 * *$ & $-19.80 * *$ & $23.10 * *$ & $30.32 * *$ & 4.23 & $9.98 *$ & $46.67 * *$ & $62.32 * *$ \\
\hline 58 & SRT-1B X R-53 & $45.23 * *$ & -1.49 & $51.19 * *$ & $60.06 * *$ & 1.53 & -1.08 & $31.91 * *$ & $46.00 * *$ \\
\hline 59 & SRT-1B X AKH-07R & $160.50 * *$ & 0.16 & $53.73 * *$ & $62.75 * *$ & $18.09 * *$ & $9.98 *$ & $46.67 * *$ & $62.32 * *$ \\
\hline \multirow[t]{3}{*}{60} & SRT-1B X DHY-286-1R & $53.90 * *$ & $-40.83 * *$ & -9.18 & -3.85 & $10.18 *$ & 6.15 & $41.56 * *$ & $56.67 * *$ \\
\hline & S.E. \pm & 4.48 & 4.48 & 4.48 & 4.48 & 0.32 & 0.32 & 0.32 & 0.32 \\
\hline & C.D. @ 5\% & 8.84 & 8.84 & 8.84 & 8.84 & 0.63 & 0.63 & 0.63 & 0.63 \\
\hline
\end{tabular}


Table.1 Continue...

\begin{tabular}{|c|c|c|c|c|c|c|c|c|c|}
\hline \multirow{2}{*}{$\begin{array}{l}\text { Sr. } \\
\text { No. }\end{array}$} & \multirow[t]{2}{*}{ Crosses } & \multicolumn{4}{|c|}{ Lint index (g) } & \multicolumn{4}{|c|}{ Harvest index (\%) } \\
\hline & & ВPH & SH 1 & SH 2 & SH 3 & ВPH & SH 1 & SH 2 & SH 3 \\
\hline 1 & CAK 53A X R-42-8 & -8.67 & 4.54 & $16.94 *$ & $16.63 *$ & $-16.31 * *$ & $-17.76 * *$ & $-16.10 * *$ & $-11.47 * *$ \\
\hline 2 & CAK 53A X R-2000-23 & 11.8 & $17.10 *$ & $30.99 * *$ & $30.64 * *$ & $-26.71 * *$ & $-33.50 * *$ & $-32.16 * *$ & $-28.42 * *$ \\
\hline 3 & CAK 53A X R- 2000-17-2 & -8.65 & 6.46 & $19.08 *$ & $18.76 *$ & -4.27 & $-17.04 * *$ & $-15.37 * *$ & $-10.71 * *$ \\
\hline 4 & CAK 53A X R-2000-26 & 10.7 & $29.45 * *$ & $44.81 * *$ & $44.42 * *$ & 3.03 & $-14.86 * *$ & $-13.14 * *$ & $-8.35 * *$ \\
\hline 5 & CAK 53A X R-1044-13 & 2.04 & 11.76 & $25.01 * *$ & $24.68 * *$ & $-24.81 * *$ & $-41.22 * *$ & $-40.04 * *$ & $-36.73 * *$ \\
\hline 6 & CAK 53A X R-2000-21 & -0.80 & 14.39 & $27.95 * *$ & $27.61 * *$ & 4.31 & $-11.61 * *$ & $-9.82 * *$ & -4.85 \\
\hline 7 & CAK 53A X R-23 & $-14.01 *$ & 8.61 & $21.49 * *$ & $21.16 *$ & $-32.05 * *$ & $-34.37 * *$ & $-33.05 * *$ & $-29.36 * *$ \\
\hline 8 & CAK 53A X R-53 & 1.12 & $15.46 *$ & $29.16 * *$ & $28.81 * *$ & 4.96 & $-13.49 * *$ & $-11.74 * *$ & $-6.88 *$ \\
\hline 9 & CAK 53A X AKH-07R & $18.23 * *$ & $45.28 * *$ & $62.51 * *$ & $62.07 * *$ & $34.04 * *$ & $15.45 * *$ & $17.77 * *$ & $24.26 * *$ \\
\hline 10 & CAK 53A X DHY-286-1R & $18.24 *$ & $17.82 *$ & $31.79 * *$ & $31.44 * *$ & $-8.74 *$ & $-37.10 * *$ & $-35.83 * *$ & $-32.30 * *$ \\
\hline 11 & CAK 53B X R-42-8 & -1.57 & 12.67 & $26.04 * *$ & $25.70 * *$ & $-21.26 * *$ & $-22.62 * *$ & $-21.06 * *$ & $-16.71 * *$ \\
\hline 12 & CAK 53B X R-2000-23 & 1.48 & 6.30 & $18.90 *$ & $18.59 *$ & -4.94 & $-13.74 * *$ & $-12.00 * *$ & $-7.15 * *$ \\
\hline 13 & CAK 53B X R- 2000-17-2 & 2.05 & $18.93 *$ & $33.04 * *$ & $32.68 * *$ & 3.24 & $-10.54 * *$ & $-8.73 * *$ & -3.7 \\
\hline 14 & CAK 53B X R-2000-26 & 8.01 & $26.31 * *$ & $41.28 * *$ & $40.91 * *$ & -3.16 & $-19.97 * *$ & $-18.36 * *$ & $-13.86 * *$ \\
\hline 15 & CAK 53B X R-1044-13 & -1.78 & 7.57 & $20.33 *$ & $20.01 *$ & $-7.62 *$ & $-27.78 * *$ & $-26.33 * *$ & $-22.27 * *$ \\
\hline 16 & CAK 53B X R-2000-21 & 9.16 & $25.87 * *$ & $40.79 * *$ & $40.42 * *$ & $-17.73 * *$ & $-30.28 * *$ & $-28.88 * *$ & $-24.96 * *$ \\
\hline 17 & CAK 53B X R-23 & $-14.80 *$ & 7.61 & $20.37 *$ & $20.05 *$ & $-23.46 * *$ & $-26.07 * *$ & $-24.58 * *$ & $-20.42 * *$ \\
\hline 18 & CAK 53B X R-53 & 8.83 & $24.27 * *$ & $39.01 * *$ & $38.64 * *$ & $22.59 * *$ & 1.04 & 3.08 & $8.76 * *$ \\
\hline 19 & CAK 53B X AKH-07R & -7.40 & 13.79 & $27.28 * *$ & $26.95 * *$ & 4.89 & $-9.66 * *$ & $-7.84 * *$ & -2.76 \\
\hline 20 & CAK 53B X DHY-286-1R & $23.28 * *$ & $22.84 * *$ & $37.41 * *$ & $37.04 * *$ & $20.49 * *$ & $-16.96 * *$ & $-15.28 * *$ & $-10.62 * *$ \\
\hline 21 & CAK 23A X R-42-8 & -1.93 & $19.77 * *$ & $33.97 * *$ & $33.61 * *$ & $-16.55 * *$ & $-17.99 * *$ & $-16.34 * *$ & $-11.72 * *$ \\
\hline 22 & CAK 23A X R-2000-23 & -6.49 & 14.19 & $27.73 * *$ & $27.39 * *$ & $-16.02 * *$ & $-23.80 * *$ & $-22.26 * *$ & $-17.97 * *$ \\
\hline 23 & CAK 23A X R- 2000-17-2 & -4.83 & $16.22 *$ & $30.00 * *$ & $29.66 * *$ & -4.10 & $-16.90 * *$ & $-15.22 * *$ & $-10.55 * *$ \\
\hline 24 & CAK 23A X R-2000-26 & 5.84 & $29.25 * *$ & $44.58 * *$ & $44.20 * *$ & $8.56 * *$ & $-10.29 * *$ & $-8.48 * *$ & -3.43 \\
\hline 25 & CAK 23A X R-1044-13 & -9.46 & 10.56 & $23.67 * *$ & $23.34 * *$ & $7.99 *$ & $-15.58 * *$ & $-13.88 * *$ & $-9.13 * *$ \\
\hline 26 & CAK 23A X R-2000-21 & -10.02 & 9.88 & $22.92 * *$ & $22.59 * *$ & -3.59 & $-18.31 * *$ & $-16.66 * *$ & $-12.06 * *$ \\
\hline 27 & CAK 23A X R-23 & $-12.87 *$ & 10.04 & $23.09 * *$ & $22.77 * *$ & $-22.71 * *$ & $-25.35 * *$ & $-23.84 * *$ & $-19.65 * *$ \\
\hline 28 & CAK 23A X R-53 & $-16.51 * *$ & 1.95 & 14.04 & 13.74 & $-7.95 * *$ & $-24.13 * *$ & $-22.60 * *$ & $-18.34 * *$ \\
\hline 29 & CAK 23A X AKH-07R & -5.48 & $16.14 *$ & $29.92 * *$ & $29.57 * *$ & $-13.31 * *$ & $-25.34 * *$ & $-23.83 * *$ & $-19.64 * *$ \\
\hline 30 & CAK 23A X DHY-286-1R & -0.46 & $21.56 * *$ & $35.98 * *$ & $35.62 * *$ & $26.04 * *$ & $-13.14 * *$ & $-11.38 * *$ & $-6.50 *$ \\
\hline 31 & CAK 23B X R-42-8 & 3.98 & $26.98 * *$ & $42.04 * *$ & $41.66 * *$ & $-9.05 * *$ & $-10.62 * *$ & $-8.81 * *$ & -3.79 \\
\hline
\end{tabular}


Table.1 Continue...

\begin{tabular}{|c|c|c|c|c|c|c|c|c|c|}
\hline \multirow{2}{*}{$\begin{array}{l}\text { Sr. } \\
\text { No. }\end{array}$} & \multirow[t]{2}{*}{ Crosses } & \multicolumn{4}{|c|}{ Lint index (g) } & \multicolumn{4}{|c|}{ Harvest index (\%) } \\
\hline & & ВPH & SH 1 & SH 2 & SH 3 & ВPH & SH 1 & SH 2 & SH 3 \\
\hline 32 & CAK 23B X R-2000-23 & 8.19 & $32.12 * *$ & $47.79 * *$ & $47.40 * *$ & $17.70 * *$ & $6.80 * *$ & $8.95 * *$ & $14.96 * *$ \\
\hline 33 & CAK 23B X R- 2000-17-2 & -9.43 & 10.6 & $23.72 * *$ & $23.39 * *$ & -1.47 & $-14.62 * *$ & $-12.90 * *$ & $-8.10 * *$ \\
\hline 34 & CAK 23B X R-2000-26 & -3.92 & $17.34 *$ & $31.25 * *$ & $30.90 * *$ & $9.48 * *$ & $-9.52 * *$ & $-7.70 * *$ & -2.61 \\
\hline 35 & CAK 23B X R-1044-13 & -0.82 & $21.12 * *$ & $35.49 * *$ & $35.13 * *$ & $14.56 * *$ & $-10.44 * *$ & $-8.64 * *$ & -3.60 \\
\hline 36 & CAK 23B X R-2000-21 & -8.13 & 12.2 & $25.50 * *$ & $25.17 * *$ & $12.86 * *$ & -4.36 & -2.43 & 2.94 \\
\hline 37 & CAK 23B X R-23 & -4.92 & $20.09 * *$ & $34.33 * *$ & $33.97 * *$ & $-6.58 *$ & $-9.77 * *$ & $-7.95 * *$ & -2.88 \\
\hline 38 & CAK 23B X R-53 & -9.92 & 10.00 & $23.05 * *$ & $22.72 * *$ & $10.75 * *$ & $-8.71 * *$ & $-6.87 * *$ & -1.74 \\
\hline 39 & CAK 23B X AKH-07R & $13.40 *$ & $39.34 * *$ & $55.86 * *$ & $55.45 * *$ & $27.38 * *$ & $9.71 * *$ & $11.92 * *$ & $18.09 * *$ \\
\hline 40 & CAK 23B X DHY-286-1R & $13.58 *$ & $38.70 * *$ & $55.15 * *$ & $54.74 * *$ & $57.42 * *$ & $8.49 * *$ & $10.68 * *$ & $16.78 * *$ \\
\hline 41 & SRT-1A X R-42-8 & 3.97 & $19.01 *$ & $33.13 * *$ & $32.77 * *$ & $-19.71 * *$ & $-21.09 * *$ & $-19.50 * *$ & $-15.07 * *$ \\
\hline 42 & SRT-1A X R-2000-23 & 6.74 & $19.29 * *$ & $33.44 * *$ & $33.08 * *$ & 0.90 & $-8.44 * *$ & $-6.60 *$ & -1.45 \\
\hline 43 & SRT-1A X R- 2000-17-2 & 4.41 & $21.68 * *$ & $36.11 * *$ & $35.75 * *$ & $-18.50 * *$ & $-26.63 * *$ & $-25.15 * *$ & $-21.02 * *$ \\
\hline 44 & SRT-1A X R-2000-26 & $17.83 * *$ & $37.78 * *$ & $54.12 * *$ & $53.71 * *$ & $18.41 * *$ & $6.60 * *$ & $8.75 * *$ & $14.74 * *$ \\
\hline 45 & SRT-1A X R-1044-13 & -1.39 & 10.2 & $23.27 * *$ & $22.94 * *$ & 3.13 & $-7.16 * *$ & $-5.29 *$ & -0.07 \\
\hline 46 & SRT-1A X R-2000-21 & 2.49 & $18.17 *$ & $32.19 * *$ & $31.84 * *$ & $-13.66 * *$ & $-22.28 * *$ & $-20.71 * *$ & $-16.34 * *$ \\
\hline 47 & SRT-1A X R-23 & -5.68 & $19.13 *$ & $33.26 * *$ & $32.90 * *$ & $-36.53 * *$ & $-38.70 * *$ & $-37.46 * *$ & $-34.02 * *$ \\
\hline 48 & SRT-1A X R-53 & 6.07 & $21.12 * *$ & $35.49 * *$ & $35.13 * *$ & $-24.35 * *$ & $-31.90 * *$ & $-30.53 * *$ & $-26.70 * *$ \\
\hline 49 & SRT-1A X AKH-07R & -8.08 & 12.95 & $26.35 * *$ & $26.01 * *$ & -2.22 & $-11.98 * *$ & $-10.21 * *$ & -5.26 \\
\hline 50 & SRT-1A X DHY-286-1R & 5.35 & $17.74 *$ & $31.70 * *$ & $31.35 * *$ & $-26.33 * *$ & $-33.68 * *$ & $-32.35 * *$ & $-28.62 * *$ \\
\hline 51 & SRT-1B X R-42-8 & 8.15 & $23.79 * *$ & $38.48 * *$ & $38.11 * *$ & $-15.35 * *$ & $-16.81 * *$ & $-15.13 * *$ & $-10.46 * *$ \\
\hline 52 & SRT-1B X R-2000-23 & $14.16 *$ & $27.58 * *$ & $42.71 * *$ & $42.33 * *$ & $12.82 * *$ & 2.37 & 4.43 & $10.19 * *$ \\
\hline 53 & SRT-1B X R- 2000-17-2 & 1.81 & $18.65 *$ & $32.72 * *$ & $32.37 * *$ & $-17.50 * *$ & $-25.73 * *$ & $-24.23 * *$ & $-20.06 * *$ \\
\hline 54 & SRT-1B X R-2000-26 & 8.28 & $26.62 * *$ & $41.64 * *$ & $41.26 * *$ & $-11.46 * *$ & $-20.29 * *$ & $-18.69 * *$ & $-14.21 * *$ \\
\hline 55 & SRT-1B X R-1044-13 & 7.49 & $20.13 * *$ & $34.37 * *$ & $34.02 * *$ & 1.83 & $-8.33 * *$ & $-6.48 *$ & -1.33 \\
\hline 56 & SRT-1B X R-2000-21 & 5.70 & $21.88 * *$ & $36.34 * *$ & $35.97 * *$ & $-14.19 * *$ & $-22.75 * *$ & $-21.19 * *$ & $-16.85 * *$ \\
\hline 57 & SRT-1B X R-23 & -2.27 & $23.44 * *$ & $38.07 * *$ & $37.71 * *$ & $-17.43 * *$ & $-20.25 * *$ & $-18.65 * *$ & $-14.16 * *$ \\
\hline 58 & SRT-1B X R-53 & 5.93 & $20.96 * *$ & $35.31 * *$ & $34.95 * *$ & $-11.01 * *$ & $-19.89 * *$ & $-18.27 * *$ & $-13.77 * *$ \\
\hline 59 & SRT-1B X AKH-07R & -5.71 & $15.86 *$ & $29.60 * *$ & $29.26 * *$ & $-6.40 *$ & $-15.74 * *$ & $-14.04 * *$ & $-9.30 * *$ \\
\hline 60 & SRT-1B X DHY-286-1R & 5.67 & $18.09 *$ & $32.10 * *$ & $31.75 * *$ & $-17.21 * *$ & $-25.47 * *$ & $-23.97 * *$ & $-19.78 * *$ \\
\hline & S.E. \pm & 0.31 & 0.31 & 0.31 & 0.31 & 0.93 & 0.93 & 0.93 & 0.93 \\
\hline & C.D. @ 5\% & 0.61 & 0.61 & 0.61 & 0.61 & 1.83 & 1.83 & 1.83 & 1.83 \\
\hline
\end{tabular}


By comparing both the sets by using different parameters based on CMS system and conventional method revealed that three crosses i.e. CAK 53 x R-2000-26, CAK 53 x R-2000-17-2 and CAK 53 x R-53 were common for $50 \%$ flowering both in CMS and conventional system.

From overall results the mean performance and heterotic expression of conventional hybrids were better than hybrid based on the CMS. The presence of the strong sterile cytoplasm have a tendency to reduce heterosis, since in such cases restorers can only restore fertility but cannot neutralize completely the adverse effect of strong sterile cytoplasm on heterosis as observed by Bhale and Bhatt (1990). However, certain cross combinations particularly CMS based showed significant high heterotic effect and better performance over the conventional hybrids. For example the cross CAK 53A X AKH -07 $\mathrm{R}$ showed heterotic effect for seed cotton yield per plant, number of sympodia per plant, number of bolls per plant and boll weight and CAK 53A X R-2000-26 for 50\% flowering depicted high mean performance and high heterotic effect compared to their counterpart based on the conventional method. This indicated that cytoplasmic male sterility could be successfully utilized for commercial exploitation of heterosis.

The crosses, CAK 53A x AKH- 07R, CAK 23B x AKH-07R, CAK 23B x DHy-286-1R, CAK 23B x R-2000-23 and SRT-1A x R2000-26 were found better for seed cotton yield per plant and hold promise for further evaluation and commercial exploitation of heterosis both in CMS and conventional system for high yield.

\section{References}

Bhale, N. L. and Bhat, M. G. 1990.Investigation on exploitation of heterosis in cotton ( $G$. huirsutumL.) using male sterility. Indian J. Genet., 50(1): 3744

Deosarkar, D. B., Jadhav D. S. and Patil S. G. 2009. Heterosis study in Cotton (Gossypium hirsutum L.) under rainfed conditions. J. Cotton Res. Dev. 23(1): 3640.

Meshram, L. D.; Wadodkar, M. B.; Kukade, B. K. and Marawar, M. W. 1995.A new source of cytoplasmic male sterility in upland cotton. Hybrid Cotton Newsletter, 3(2): 3 .

Patel, D. H., Patel, D. U. and Kumar, V. 2014.Hetrosis and combining ability analysis in tetrapoloid cotton (G.hirsutum and G.barbadense). Electronic J. of Plant Breeding, 5(3): 408-414.

Pushpam, R., Thangaraj, K. and Raveerandran, T. S. 2015. Heterosis and combining ability studies in upland cotton for yield characters. Electronic Journal of Plant Breeding. 6(2): 459-463.

Sharma, R., Gill, B. S., and Pathak, D. 2016.Hetrobeltiosis for yield, its component traits and fibre properties in upland cotton (Gossypium hirsutum). J. Cotton Res. Dev. 30 (1) 11-15.

Shinde, G. C., Mehetre, S. S. and Jagtap, P. K. 2012 Combining ability and heterosis for seed cotton yield and its component traits in CGMS based hybrids of cotton (G. hirsutum L) cotton Research Journal. Jan.-Jun (2012) 23-32.

Tuteja, O. P., Verma, S. K. and Banga, M. 2011. Heterosis for seed cotton yield and other traits in GMS (Genetic male sterility) based hybrids of Gossypium hirsutum L cotton. J. Cotton Res. Dev. 25(1): 14-18.

Tuteja, O. P. and Agrawal, M. 2014. Hetrosis for seed cotton yield and other traits in GMS based hybrids of American cotton (G. hirsutum.). Cotton Research J. 5 (2): 131-141. 


\section{How to cite this article:}

Gopal, G. R., D. B. Deosarkar and Chinchane, V. N. 2020. Heterosis Studies in CMS Based and Conventional Hybrids for Yield and Yield Contributing Traits in Cotton (G. hirsutum L.) Over the Environments. Int.J.Curr.Microbiol.App.Sci. 9(03): 1212-1227. doi: https://doi.org/10.20546/ijcmas.2020.903.142 